\title{
Tendencia de la Dinámica No-Lineal en una Precontingencia Ambiental causada por Partículas en Suspensión
}

\author{
Estela Audelo-Vucovich(1), Carlos Vázquez-Cruz(1) y Fernando Beristain ${ }^{(2)}$ \\ (1) Centro de Cómputo, (2) Departamento de Ingeniería y Tecnología (LAPRyFAL) \\ Facultad de Estudios Superiores Cuautitlán, Universidad Nacional Autónoma de México, Av. $1^{\circ}$ de mayo \\ S/N Colonia Santa María las Torres, C.P. 54740, Cuautitlán Izcalli, Estado de México. México. \\ (e-mail: eavucovich@gmail.com; carlos@unam.mx; ferber@unam.mx)
}

Recibido Dic. 18, 2014; Aceptado Mar. 4, 2015; Versión final Abr. 27, 2015, Publicado Ago. 2015

\begin{abstract}
Resumen
Se encontró la tendencia de los índices de la dinámica no lineal en una precontingencia ambiental por PM10 en la Zona Metropolitana del Valle de México. Se aplicaron las herramientas de la teoría del caos, para determinar las coincidencias en su comportamiento dinámico y se seleccionó el periodo de estudio del 15 de diciembre al 7 de enero. Se calcularon los índices de la dinámica no lineal para evaluar los cambios en el comportamiento dinámico y se graficó el atractor en el espacio de fases. Los resultados obtenidos en cuanto a persistencia, divergencia y autocorrelación varían dependiendo de la concentración de PM10. El exponente de Hurst permitió encontrar la mejor concordancia con las partículas $\mathrm{PM}_{10}$ y se observó que al superar el valor de 0.3 aumenta la probabilidad de alcanzar un evento de precontingencia.
\end{abstract}

\section{Trend of Nonlinear Dynamics in an Environmental Pre-contingency due to Particles in Suspension}

\begin{abstract}
The trend of the indexes of the nonlinear dynamics in an environmental pre-contingency by particles $\mathrm{PM}_{10}$ in the Metropolitan Area of Mexico Valley was found. The tools of chaos theory were applied to determine the dynamic behavior coincidences and the study period from December 15 to January 7 was selected. The indexes of nonlinear dynamics were calculated to evaluate the changes in the dynamic behavior and the attractors in the phase space were plotted. The results obtained in terms of persistence, divergence and autocorrelation vary depending on the concentration of PM10. The Hurst exponent allowed finding the best concordance with $\mathrm{PM}_{10}$ and it was observed that when it exceeds the value of 0.3 the probability of reaching a pre-contingency event increases.
\end{abstract}

Keywords: environmental pre-contingency, PM10 particles, nonlinear dynamics, chaos theory 


\section{INTRODUCCIÓN}

La contaminación del aire representa un importante riesgo medioambiental para la salud, bien sea en los países desarrollados o en los países en desarrollo. Las Directrices de la Organización Mundial de la Salud (OMS, 2005) sobre la Calidad del Aire publicadas en 2005 ofrecen orientaciones generales relativas a umbrales y límites para contaminantes de la atmósfera que entrañan riesgos sanitarios; se aplican en todo el mundo y se basan en la evaluación realizada por expertos. Generalmente, las mediciones de la calidad del aire se notifican como concentraciones medias diarias o anuales de partículas con diámetro aerodinámico menor a 10 micrómetros (PM10) por metro cúbico $\left(\mathrm{m}^{3}\right)$ de aire. Los valores fijados en las directrices de la OMS para PM10, se establecen en $20 \mu \mathrm{g} / \mathrm{m}^{3}$ de media anual y $50 \mu \mathrm{g} / \mathrm{m}^{3}$ de media en 24 horas. La normatividad federal estadounidense para el promedio de 24 horas de $\mathrm{PM}_{10}$ establece un límite de $150 \mu \mathrm{g} / \mathrm{m}^{3}$ (EPA, 2012). La normatividad mexicana para las $\mathrm{PM}_{10}$ es de $40 \mu \mathrm{g} / \mathrm{m}^{3}$ de media anual y $75 \mu \mathrm{g} / \mathrm{m}^{3}$ de media en 24 horas (NOM 025-SSA1-2014). Las normas nacionales varían en función del enfoque adoptado con el fin de equilibrar los riesgos para la salud, la viabilidad tecnológica, los aspectos económicos y otros factores políticos y sociales.

Entre los constituyentes de la atmosfera el material particulado, término aplicado para las partículas sólidas o liquidas encontradas en el aire, es único en complejidad; este tipo de contaminante es el resultado no solamente de la emisión directa de las partículas sino también de ciertos gases que se condensan en forma de partículas directamente o sufren una transformación química (Rojano et al., 2014; Celis et al., 2007), así, se origina de una gran variedad de fuentes móviles y fijas por lo que su composición química y física varía ampliamente (Pérez-Vidal et al., 2010; Meza et al., 2010). La determinación de los niveles de material particulado en la atmósfera es uno de los parámetros fundamentales en el control de la calidad del aire, como consecuencia de sus efectos nocivos sobre la salud, el clima y los ecosistemas (Rojano et al., 2013; Oyarzun, 2010). De los efectos agudos, se ha identificado que el incremento en la concentración de PM10 y ozono se encuentran relacionadas con un incremento en la tasa de mortalidad prematura (O'Neill et al., 2004) y en incidencia de morbilidad por infecciones respiratorias agudas (Ramírez-Rembao, 2009); en las Directrices de la OMS de 2014, sobre la calidad del aire se estima que una reducción media anual de las concentraciones de partículas (PM10) de $70 \mu \mathrm{g} / \mathrm{m}^{3}$, común en muchas ciudades en desarrollo, a $20 \mu \mathrm{g} / \mathrm{m}^{3}$, permitiría reducir el número de defunciones relacionadas con la contaminación en aproximadamente un $15 \%$.

La contingencia ambiental supone un episodio de contaminación severa, durante el cual, las concentraciones de ozono o de partículas suspendidas alcanzan niveles que ponen en riesgo la salud de la población en general. El Índice Metropolitano de la Calidad del Aire (IMECA) funciona como un valor de referencia. La Dirección de Monitoreo Atmosférico de la Zona Metropolitana del Valle de México (ZMVM), en el Programa para Contingencias Ambientales establece que la precontingencia ambiental se activa para PM10 cuando los valores del IMECA rebasan los 150 puntos (Tabla 1). En la tabla 2 se muestran los algoritmos para calcular los puntos IMECA en base a la concentración de PM10 en $\mu \mathrm{g} / \mathrm{m}^{3}$.

Tabla 1: Categorías de calidad del aire indicadas por la concentración de PM10 (Promedio de 24 hrs.) y el IMECA. Modificado de Índice de Calidad del Aire (2014); http://www.aire.df.gob.mx/.

\begin{tabular}{|l|c|c|c|c|c|}
\hline Calidad del Aire & Buena & Regular & Mala & Muy Mala & $\begin{array}{c}\text { Extremadamente } \\
\text { Mala }\end{array}$ \\
\hline IMECA (Límites) & $0-50$ & $51-100$ & $101-150$ & $151-200$ & $>200$ \\
\hline PM10 $\left(\mu \mathrm{g} / \mathrm{m}^{3}\right)$ & $0-60$ & $61-120$ & $121-220$ & $221-320$ & $>320$ \\
\hline
\end{tabular}

Tabla 2: Algoritmo de cálculo del IMECA para PM10 en la Zona Metropolitana del Valle de México

\begin{tabular}{|c|c|}
\hline Límites PM10 $\left(\mu \mathrm{g} / \mathrm{m}^{3}\right)$ & Cálculo del IMECA para PM10 \\
\hline 0 a 120 & IMECA PM10 $=$ concentración de PM10 *0.833 \\
\hline 121 a 320 & IMECA PM10 $=($ concentración de PM10 * 0.5) +40 \\
\hline Mayor a 320 & IMECA PM10 $=$ concentración de PM10 * 0.625 \\
\hline
\end{tabular}


Las herramientas de la dinámica no lineal se han empleado para caracterizar la contaminación del aire en diferentes países: en zonas rurales de California, analizando cuatro diferentes tipos de calidades del aire diarias, encontrando la presencia de un comportamiento determinista de baja dimensión (Sivakumar et al., 2007), en México se caracterizó el comportamiento de la contaminación ambiental encontrando que depende del día, hora, estación del año y tipo de uso de suelo (Vázquez et al., 2012); analizando los resultados de algunos índices de la dinámica no lineal de series de tiempo de contaminación del aire en Taiwán, se encontró comportamiento caótico con valores de entropía de Kolmogorov positiva, dimensión de correlación baja y valores de exponente de Lyapunov positivos (Lee and Lin, 2008). En el estudio de sistemas dinámicos el primer paso es la obtención y preparación de datos experimentales para el análisis; parte significativa de esto, es la elección del lugar y método de muestreo (Rojano et al., 2013), además, el filtrado y los esquemas de discretización de los datos son importantes ya que una elección inadecuada puede producir falsos resultados (Rafti et al., 2010); el segundo paso es la determinación de índices y gráficos en el espacio de fases (atractor).

Los sistemas dinámicos se caracterizan por evolucionar con el tiempo, presentando características típicas de su comportamiento, ya sea mediante índices o las referidas a la geometría que caracteriza su espacio de estados (Navarro, 2010); en un estudio realizado por Cross y Gilmore (2009), se demostró que la estructura del atractor depende de la dimensión de incrustación ya que se generan distintas representaciones con diferentes dimensiones de incrustación; por otro lado, en flujos de tráfico en redes de computadora se obtuvo un nuevo modelo multifractal, con el cual se explicó el fenómeno de localidad que aparece en la estimación del exponente de Hurst en series temporales (Millán y San Juan, 2013). En el presente trabajo, se propone calcular los índices de la dinámica no lineal antes y durante una precontingencia ambiental por PM10 y encontrar las coincidencias en la tendencia con el comportamiento de las partículas en la Zona Metropolitana del Valle de México, aplicando las herramientas de la teoría del caos y determinando las variaciones en su comportamiento dinámico.

\section{MATERIALES Y MÉTODOS}

La zona de estudio comprende la Zona Metropolitana del Valle de México (ZMVM), está conformada por el Distrito Federal y 60 municipios conurbados. La zona cuenta con una población aproximada de 20 millones de habitantes, se encuentra a $2240 \mathrm{~m}$ sobre el nivel del mar, la temperatura media anual es de $16{ }^{\circ} \mathrm{C}$ y la precipitación total anual es de $600 \mathrm{~mm}$ a $1200 \mathrm{~mm}$ (ver figura 1).

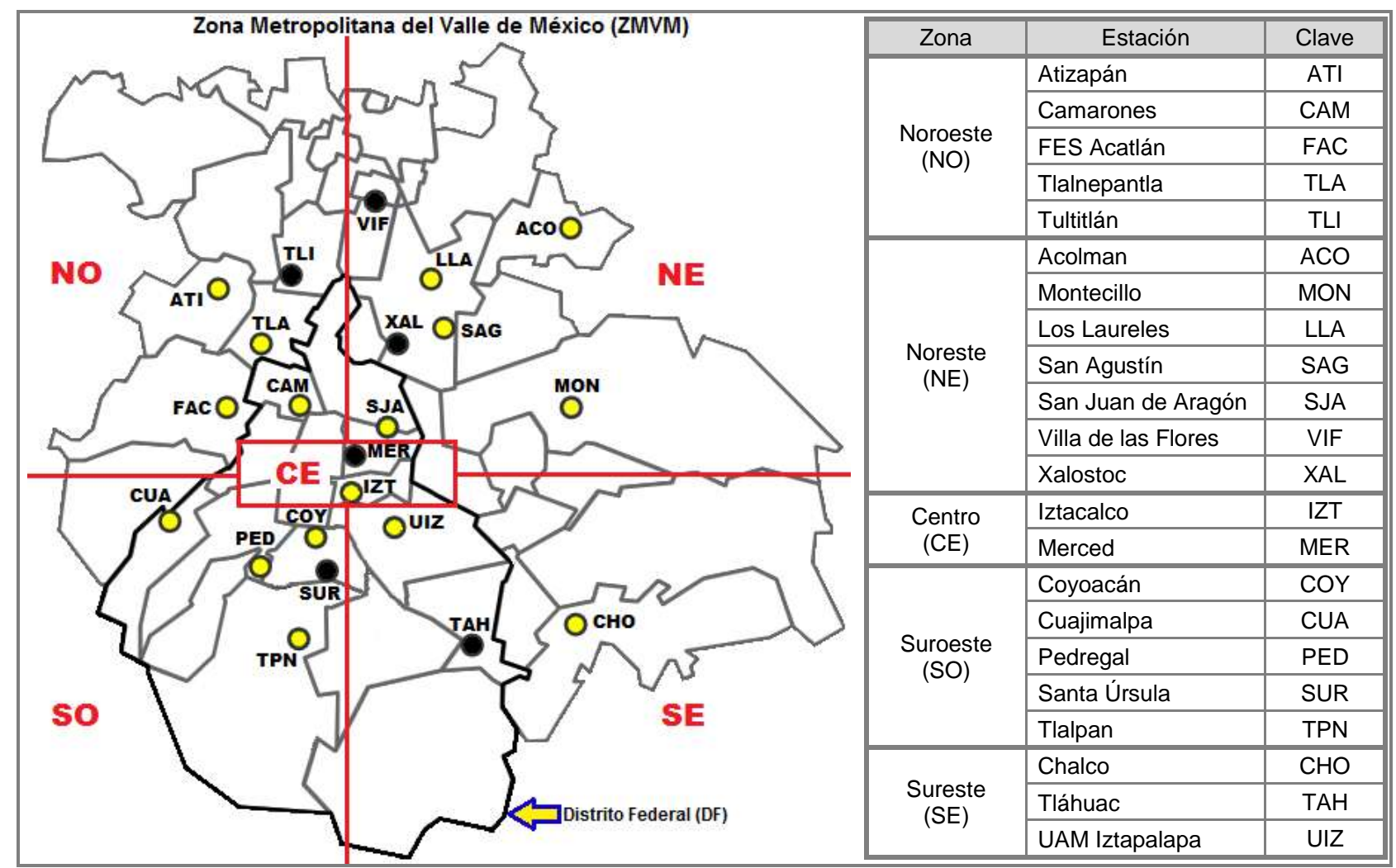

Fig. 1: Mapa de localización de las estaciones de monitoreo ambiental activas que miden $\mathrm{PM}_{10}$ de la Red Automática de Monitoreo Atmosférico en la ZMVM. En negro se observan las estaciones en estudio. Modificado de la Dirección de Monitoreo Atmosférico; http://www.aire.df.gob.mx/. 
Del histórico de precontingencias ambientales por PM10 de la ZMVM, se seleccionaron dos periodos donde se activo la fase de precontingencia ambiental por PM10, cuyas fechas fueron del 25 al 26 de diciembre del 2012 y del 1 al 2 de enero del 2013; dichas precontingencias se declararon en la zona NorEste (NE); encontrando que la concentración de $\mathrm{PM}_{10}$ para activar la precontingencia fue de $224 \mu \mathrm{g} / \mathrm{m}^{3}$ y de $236 \mu \mathrm{g} / \mathrm{m}^{3}$ respectivamente. De la Red Automática de Monitoreo Atmosférico se obtuvieron las bases de datos de PM10 de los años 2012 y 2013; revisando cuales estaciones estaban activas, medían PM10 y tenían la cantidad de datos suficientes para el análisis (más de 95\%); así, de la zona NorEste (NE), zona en la que se decretó la precontingencia, se seleccionaron las estaciones Villa de las Flores (VIF) y Xalostoc (XAL). Se eligieron otras cuatro estaciones de las demás zonas geográficas del área metropolitana para aplicar el análisis y observar si existe diferencia en los resultados. Las otras estaciones fueron: de la zona NorOeste (NO), Tultitlan (TLI); de la zona Centro (CE), Merced (MER); de la zona SurOeste (SO), Santa Úrsula (SUR) y de la zona SurEste (SE), Tláhuac (TAH), ver figura 1.

Para tener en el estudio valores de $\mathrm{PM}_{10}$ de antes y después de la fase de precontingencia, se consideró tomar datos desde el 15 de diciembre (dic) de 2012 hasta el 7 de enero (ene) de 2013. Se dividió el tiempo de estudio en periodos de 96 horas (número de datos suficiente para aplicar la metodología de análisis no lineal) con un desplazamiento de 24 horas, para observar la evolución del proceso de contaminación. Las series de tiempo obtenidas de los 21 periodos de 96 horas se muestran en la tabla 3 , el sombreado indica las series que contienen valores (fechas) de la precontingencia ambiental.

Tabla 3: Series de tiempo y periodos comprendidos

\begin{tabular}{|c|c|c|c|c|c|}
\hline Serie & Periodo & Serie & Periodo & Serie & Periodo \\
\hline$A$ & 15 al 18 dic & $H$ & 22 al 25 dic & 0 & 29 dic al 01 ene \\
\hline$B$ & 16 al 19 dic & I & 23 al 26 dic & $P$ & 30 dic al 02 ene \\
\hline$C$ & 17 al 20 dic & $J$ & 24 al 27 dic & $Q$ & 31 dic al 03 ene \\
\hline$D$ & 18 al $21 \mathrm{dic}$ & $K$ & 25 al 28 dic & $R$ & 01 al 04 ene \\
\hline$E$ & 19 al 22 dic & $L$ & 26 al 29 dic & $S$ & 02 al 05 ene \\
\hline$F$ & 20 al 23 dic & $M$ & 27 al 30 dic & $T$ & 03 al 06 ene \\
\hline$G$ & 21 al 24 dic & $N$ & 28 al 31 dic & $U$ & 04 al 07 ene \\
\hline
\end{tabular}

Con las 21 series de tiempo obtenidas por cada estación de monitoreo, se calcularon los índices de la dinámica no lineal para evaluar los cambios en el comportamiento dinámico. Con el exponente de Lyapunov se calculó el efecto causado por una pequeña perturbación y la divergencia entre los puntos vecinos. La pérdida de información y la autocorrelación se determinaron con la entropía de Kolmogorov-Sinai y la frecuencia dominante (método de máxima entropía). Mediante el cálculo del exponente de Hurst se obtuvo la autosemejanza o persistencia. Las dimensiones de capacidad y de correlación dieron una medida de la complejidad. Al graficar la evolución del sistema en el espacio de fases se obtuvo el atractor que muestra el cambio estructural durante el periodo de estudio.

\section{RESULTADOS Y DISCUSIÓN}

Del análisis estadístico los resultados más representativos fueron los percentiles 20 y 90 de la concentración de PM10 de las estaciones Villa de las Flores (VIF) y Xalostoc (XAL), la tendencia en estas dos estaciones es aumentar durante las etapas de precontingencia, en la estación Villa de las Flores el percentil 90 está en el límite de los $180 \mu \mathrm{g} / \mathrm{m}^{3}$, llegando a rebasar en el segundo periodo valores por arriba de los $300 \mu \mathrm{g} / \mathrm{m}^{3}$; en la estación Xalostoc, el comportamiento del contaminante en los dos periodos estudiados es semejante, aunque no alcanza los niveles de Villa de las Flores. La mediana nos indica que el $50 \%$ de los datos se encuentra por arriba de los $80 \mu \mathrm{g} / \mathrm{m}^{3}$. Asimismo, se confirma que el comportamiento del exponente de Hurst está identificado con la tendencia de la concentración del contaminante (ver figura 2). La línea que cruza a los $60 \mu \mathrm{g} / \mathrm{m}^{3}$ (límite de la buena calidad del aire) nos permite identificar que aún antes de ser decretada la precontingencia los niveles de PM10 rebasan ese límite. Las flechas de color azul y rojo indican los periodos de precontingencia (ver figura 2).

La dinámica no lineal es una herramienta de apoyo que interpreta y analiza datos en una serie de tiempo univariada para conocer su comportamiento dinámico mediante índices y gráficos en el espacio de fases. Al estudiar los resultados obtenidos, se observó que algunos índices mostraban claramente el comportamiento y tendencia de las $\mathrm{PM} 10$ en la precontingencia. 

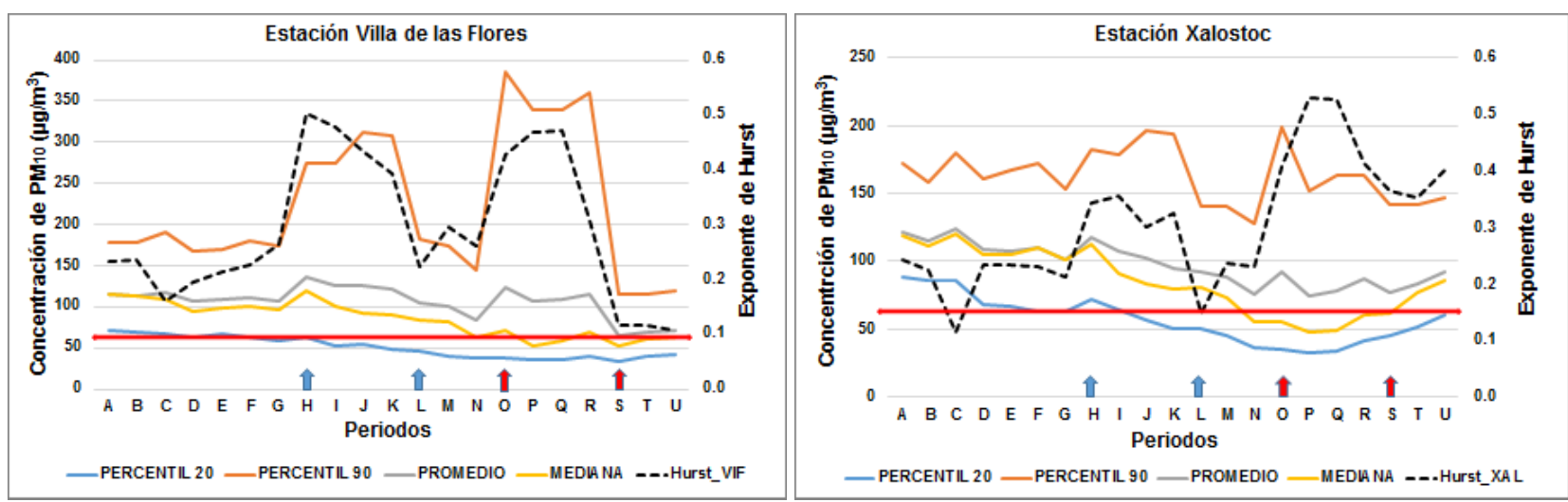

Fig. 2: Tendencia de las concentraciones de $\mathrm{PM} 10$ en las estaciones de la zona Noreste.

Uno de estos índices es el exponente de Hurst también llamado índice de la tendencia a largo plazo, el cual, dependiendo de su valor, nos dice si el sistema es autosemejante o no, al evolucionar en el tiempo. En este caso tenemos una autocorrelación negativa, es decir, que un valor alto será seguido por un valor bajo. Otro índice es el exponente de Lyapunov quie nos indica si un sistema es estable, esto es, cuando se le produce una pequeña perturbación, su trayectoria a través del espacio de fases cambia sólo un poco. Los resultados obtenidos nos hablan de un sistema dinámico no estable con un comportamiento aperiódico. Por último, la frecuencia dominante nos ayuda a eliminar tendencias en la información. Los valores encontrados permiten diferenciar las series antes y después de la precontingencia.

Con los resultados obtenidos en el análisis dinámico no lineal de cada serie de tiempo, se graficaron las tendencias del exponente de Hurst, del exponente de Lyapunov y la entropía de Kolmogorov de las estaciones en estudio, junto con la concentración promedio de PM10 de la estación Villa de las Flores (VIF) que pertenece a la zona en dónde se decretó la precontingencia ambiental; se observó, en el patrón de comportamiento del exponente de Hurst una tendencia a aumentar antes y durante la fase de precontingencia, disminuyendo conforme se llega al final del primer periodo $(\mathrm{H}-\mathrm{L})$, algo equivalente ocurre en el segundo periodo (O-S). Asimismo, se encontró que la tendencia del exponente de Hurst fue equivalente en todas las estaciones estudiadas, siguiendo el comportamiento de la concentración de PM10 (ver figura 3) independientemente de los valores de la concentración del contaminante en todas las estaciones estudiadas; asimismo, cabe hacer notar que cuando el exponente de Hurst superó el valor de 0.3, la concentración por $\mathrm{PM}_{10}$ se aproxima a los valores por los que se decretaron los eventos de precontingencia ambiental.

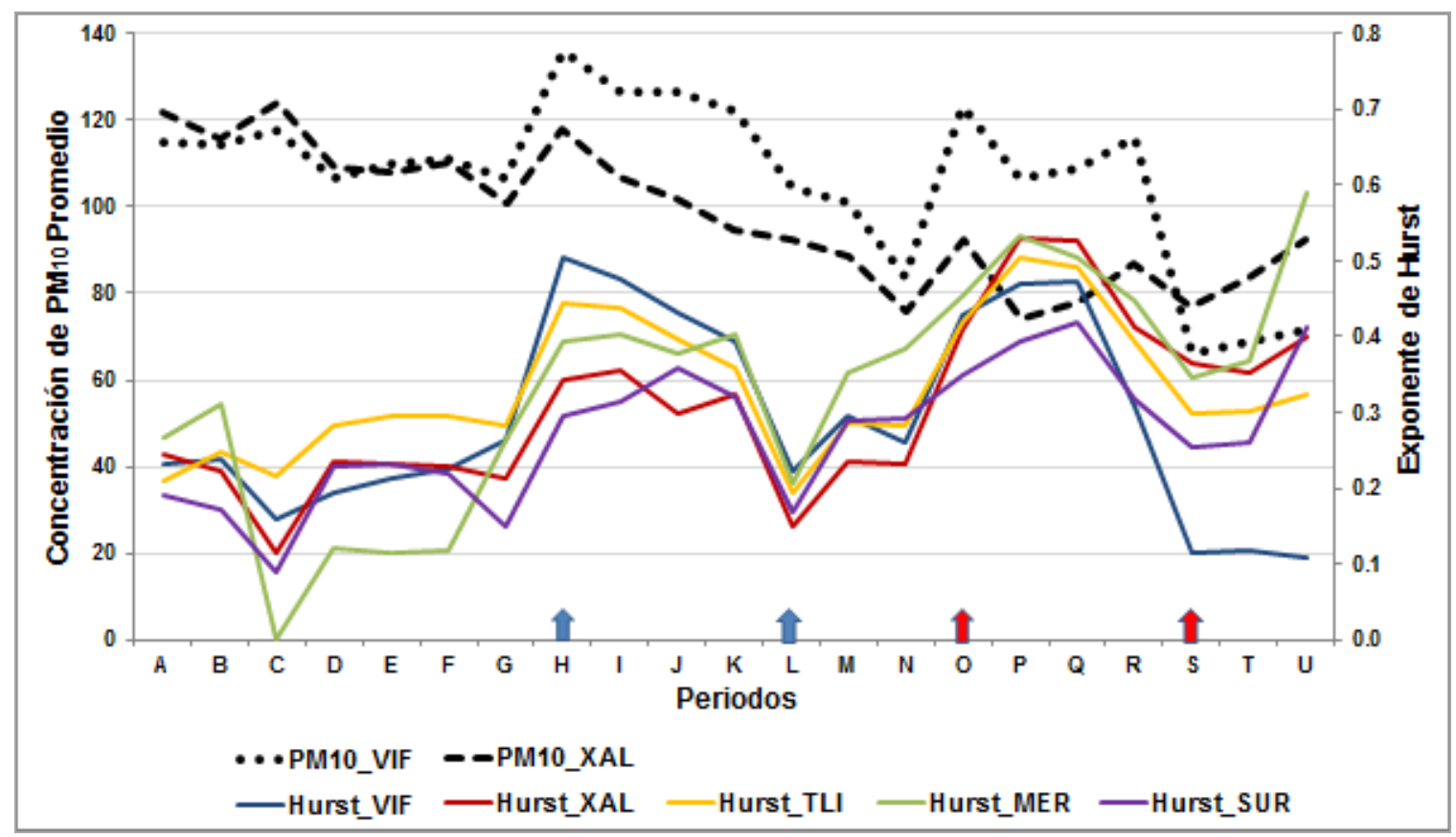

Fig. 3: Concentración de PM10 promedio y tendencias del exponente de Hurst en las estaciones en estudio. Las flechas de color indican los periodos de precontingencia. 
Gráficamente la distribución de probabilidad, el análisis de componentes principales, la entropía de kolmogorov, la dimensión de capacidad y la dimensión de correlación, no muestran un patrón de comportamiento que pueda relacionarse con el aumento de la concentración de PM10 durante la precontingencia. En la figura 4 se presentan los valores del exponente de Lyapunov, comparando dichos resultados con la concentración promedio de $\mathrm{PM}_{10}$ se observa que la divergencia del sistema disminuye cuando la concentración de PM10 aumenta y existe un intervalo, entre 0.3 y 0.5 donde es más probable que se produzca la contingencia ambiental. Los resultados del análisis espectral se muestran en la tabla 4. Cuando los valores de frecuencia dominante son mayores de 0 y menores de 0.045 la concentración de PM10 es alta.

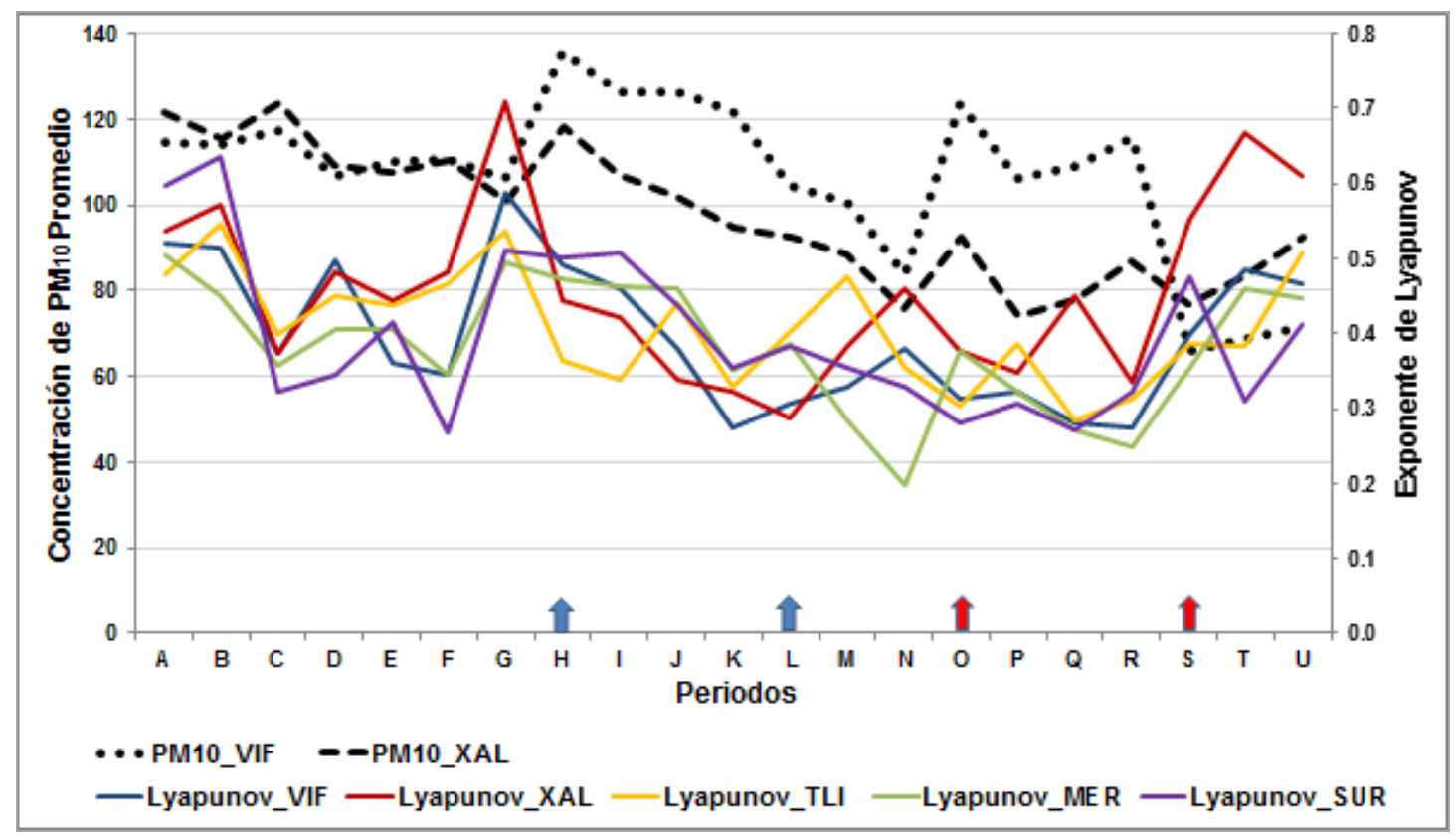

Fig. 4: Concentración de PM10 promedio y tendencias del exponente de Lyapunov en las estaciones en estudio. Las flechas de color indican los periodos de precontingencia.

Tabla 4: Promedio de PM10 y frecuencia dominante calculada en las estaciones en estudio

\begin{tabular}{|c|c|c|c|c|c|c|c|}
\hline \multirow{2}{*}{ Series } & \multirow{2}{*}{ Prom PM10 } & \multicolumn{7}{|c|}{ Frecuencia dominante } \\
\cline { 3 - 7 } & & VIF & $X A L$ & TLI & MER & SUR & TAH \\
\hline$A$ & 115 & 0.083 & 0.079 & 0.082 & 0.000 & 0.060 & 0.099 \\
\hline$B$ & 114 & 0.086 & 0.080 & 0.077 & 0.000 & 0.090 & 0.000 \\
\hline$C$ & 118 & 0.088 & 0.074 & 0.071 & 0.000 & 0.042 & 0.000 \\
\hline$D$ & 107 & 0.000 & 0.000 & 0.055 & 0.000 & 0.042 & 0.000 \\
\hline$E$ & 110 & 0.000 & 0.000 & 0.051 & 0.000 & 0.041 & 0.030 \\
\hline$F$ & 111 & 0.000 & 0.000 & 0.035 & 0.000 & 0.051 & 0.047 \\
\hline$G$ & 106 & 0.000 & 0.000 & 0.106 & 0.000 & 0.093 & 0.000 \\
\hline$H$ & 136 & 0.042 & 0.057 & 0.040 & 0.049 & 0.054 & 0.059 \\
\hline$I$ & 127 & 0.039 & 0.000 & 0.000 & 0.000 & 0.037 & 0.055 \\
\hline$J$ & 127 & 0.044 & 0.000 & 0.009 & 0.021 & 0.000 & 0.029 \\
\hline$K$ & 122 & 0.000 & 0.000 & 0.043 & 0.033 & 0.024 & 0.033 \\
\hline$L$ & 105 & 0.084 & 0.101 & 0.071 & 0.000 & 0.000 & 0.000 \\
\hline$M$ & 101 & 0.078 & 0.000 & 0.061 & 0.000 & 0.000 & 0.000 \\
\hline$N$ & 84 & 0.062 & 0.000 & 0.054 & 0.000 & 0.000 & 0.000 \\
\hline$O$ & 123 & 0.026 & 0.037 & 0.037 & 0.028 & 0.033 & 0.000 \\
\hline$P$ & 106 & 0.024 & 0.035 & 0.000 & 0.037 & 0.032 & 0.029 \\
\hline$Q$ & 109 & 0.025 & 0.037 & 0.022 & 0.039 & 0.032 & 0.000 \\
\hline$R$ & 116 & 0.000 & 0.038 & 0.030 & 0.033 & 0.030 & 0.000 \\
\hline$S$ & 66 & 0.110 & 0.034 & 0.065 & 0.000 & 0.000 & 0.000 \\
\hline$T$ & 69 & 0.087 & 0.054 & 0.062 & 0.057 & 0.000 & 0.000 \\
\hline$U$ & 72 & 0.000 & 0.046 & 0.049 & 0.042 & 0.039 & 0.063 \\
\hline
\end{tabular}


El espacio de fases es el espacio matemático constituido por las variables que describen un sistema dinámico, donde cada punto representa un posible estado del sistema. Los gráficos en el espacio de fases llamados también atractores representan la evolución en el tiempo del sistema. En la figura 5 se muestran los atractores de la estación de monitoreo Villa de las Flores, la primera fila corresponde a las series antes de la precontingencia $(E, F$ y G), y en la segunda, las series durante la precontingencia $(H, I$ y J). Se observa que durante la precontingencia tienen mayor densidad en la cuenca de atracción y las trayectorias más lejanas presentan un lazo (marcado con un círculo rojo).
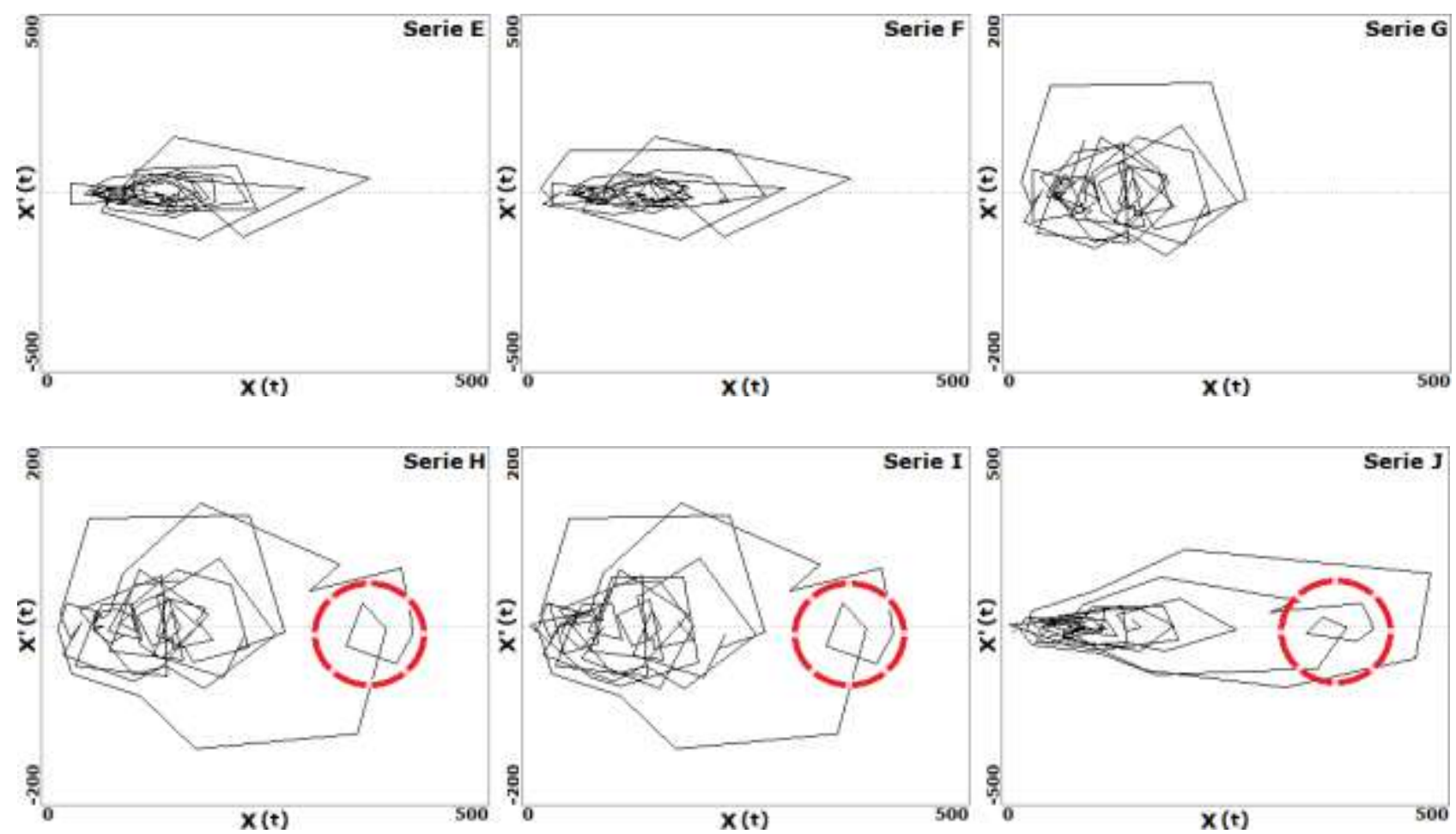

Fig. 5: Representación geométrica de las trayectorias en el espacio fase $\left(X^{\prime}(t)\right.$ vs $\left.X(t)\right)$, antes de la precontingencia series $(E, F$ y $G)$ y durante la precontingencia las series de $(H, I$ y $J)$.

\section{CONCLUSIONES}

Se determinó que el cambio en la concentración de las partículas $\mathrm{PM}_{10}$ tiene un efecto en el comportamiento dinámico no lineal. De los análisis realizados, el exponente de Hurst, el exponente de Lyapunov, la frecuencia dominante y el gráfico en el espacio de fases se seleccionaron como invariantes para diferenciar el comportamiento dinámico del sistema.

El seguimiento de los valores (cuando se supera 0.3) y del patrón de comportamiento del exponente de Hurst, junto con el cambio en la estructura del gráfico en el espacio de fases, permite determinar con anticipación la probable aparición de un evento de precontingencia ambiental. Asimismo, se encontró que existe un intervalo de valores del exponente de Lyapunov $(0.5>\lambda>0.3)$ y la frecuencia dominante $(0.045>\mathrm{fd}>0.025)$ que nos permiten reconocer que se aproxima un periodo de precontingencia. Se encontró que el seguimiento de la evolución del sistema, puede ser un método alternativo o de apoyo que nos permita distinguir las etapas de una precontingencia ambiental por PM10.

\section{REFERENCIAS}

Celis, J.E., Morales, J.R., Zaror, C.A. y Carvacho, O.F., Contaminación del Aire Atmosférico por Material Particulado en una Ciudad Intermedia: El caso de Chillan (Chile), Información Tecnológica 18(3), 49-58 (2007)

Cross, D.J. y Gilmore, R., Representation theory for strange attractors, doi: 10.1103/PhysRevE.80.056207, Physical Review (en línea) E 80, 056207 (2009)

EPA (Environmental Protection Agency), Agencia de Protección ambiental de Estados Unidos, Nuevo estándar de partículas finas (2012). http://www.epa.gov/air/particlepollution. Acceso: octubre (2014)

Lee, C.K. y Lin, S.C., Chaos in Air Pollutant Concentration (APC) Time Series, Aerosol and Air Quality Research, Vol. 8, No.4, pp. 381-391 (2008) 
Meza, L.M., Quintero, M., García, R. y Ramírez, J., Estimación de Factores de Emisión de PM10 y PM2.5, en Vías Urbanas en Mexicali, Baja California, México, Información Tecnológica Vol. 21(4), 45-56 (2010)

Millán, N.G. y San Juan, U.E., Un modelo multifractal simplificado para flujos de tráfico en redes de computadoras de alta velocidad, Ingeniare. Revista chilena de ingeniería, vol. 21 № 3, pp. 408-413 (2013)

Navarro, L.E.M., La Naturaleza, las Matemáticas, la Ingeniería y el Caos, Ingeniare. Revista chilena de ingeniería, vol. 18 № 1, pp. 5-7 (2010)

NOM-025-SSA1 (Norma Oficial Mexicana), Salud ambiental, valores límites permisibles para la concentración de partículas suspendidas PM10 y PM2.5 en el aire ambiente y criterios para su evaluación (2014)

OMS (Organización Mundial de la Salud), Guías de Calidad del aire de la OMS, relativas al material particulado, el ozono, el dióxido de nitrógeno y el dióxido de azufre. Actualización (2005)

O'Neill, M.S., Loomis, D., Borja-Aburto, V.H., Gold, D., Hertz-Picciotto, I. y Castillejos, M., Do associations between airborne particles and daily mortality in Mexico City differ by measurement method, region, or modeling strategy?, J. Exp. Anal. Environ. Epidem. 14: 429-439 (2004)

Oyarzun, M., Contaminación aérea y sus efectos en la salud, doi: 10.4067/S0717-73482010000100004, Rev. Chilena de enfermedades respiratorias (en línea), 26(1), 16-25 (2010)

Pérez-Vidal, H., Lunagómez-Rocha, Ma., Acosta-Pérez, Li., Análisis de partículas suspendidas totales (PST) y partículas fracción respirable (PM10), en Cunduacán, Tabasco, Universidad y Ciencia: 26(2), 151-162 (2010)

Rafti, M., Cordero, C. y Vicente, J.L., Influencia del Tratamiento de Datos en la Detección de Regímenes Caóticos en series Temporales, Información Tecnológica Vol. 21(4), 11-16 (2010)

Ramirez-Rembao, M., Rojas, R.I. y García-Cueto, R., Influencia de los Contaminantes Atmosféricos en las Infecciones Respiratorias Agudas en Mexicali-Baja California, México, Información Tecnológica Vol. 20(3), 89-100 (2009)

Rojano, R.E., Angulo, L.C., Restrepo, G., Niveles de Partículas Suspendidas Totales (PST), PM10 y PM2.5 y su Relación en Lugares Públicos de la Ciudad Riohacha, Caribe Colombiano, Información Tecnológica 24(2), 37-46 (2013)

Rojano, R., Arregoces, H. y Restrepo, G., Composición Elemental y Fuentes de Origen de Partículas Respirables (PM10) y Partículas Suspendidas Totales (PST) en el Área Urbana de la Ciudad de Riohacha, Colombia, doi: 10.4067/S0718-07642014000600002, Inf. Tecnol.(enlínea), 25(6), 3-12 (2014)

Sivakumar, B., Wallender, W. W., Horwath, W. R. y Mitchell, J. P., Nonlinear Deterministic Analysis of Air Pollution Dynamics in a Rural and Agricultural Setting, Advances in Complex Systems, Vol. 10, No. 4, 581597 (2007)

Vázquez, C., Beristain, F. y Audelo, E., Caracterización del Patrón de Comportamiento de las Partículas en Suspensión en el Valle de México, Información Tecnológica Vol. 23(3), 3-10 (2012) 